\title{
Comparative Therapy of Animal and Bird Diseases Caused by Mycoplasmas
}

\author{
Valery Agoltsov, Larisa Padilo, and Oksana Biryukova*
}

Federal State-Owned Publicly-Funded Institution of Higher Education Saratov State Agrarian University named after N.I. Vavilov, Saratov, 410012, Russia, 1 Teatralnaya pl.

\begin{abstract}
Mycoplasmas are the cause of many pathologies, both of various species of animals and birds. The minimum inhibitory concentrations (MICs) of enrofloxacin, difloxacin, oxytetracycline, chlortetracycline, doxycycline, tylosin, tilmycosin, tilvalosin, tiamulin, florfenicol, lincomycin, spectinomycin and 2 combinations (spectinomycin and lincomycin) with the isolates of Myecoplasma sympathy were significantly different. Elevated MICs of tilmicosin were observed in both M. synoviae and M. gallisepticum isolates (MIC values $>=64 \mu \mathrm{g} / \mathrm{ml}$ ), and this was observed in all isolates with high MICs of tylosin. The increase in the MIC of lincomycin was mainly associated with the increase in the MIC of tilmicosin. In vitro susceptibility testing of $50 \mathrm{M}$. gallisepticum strains isolated in Israel in the period of 1997-2010 carried out by a group of scientists led by Gerchman I. showed that acquired resistance to tylosin, as well as to tilmicosin, is present in $50 \%$ of them. Moreover, $72 \%(13 / 18)$ of the strains isolated from clinical specimens since 2006 showed acquired resistance to enrofloxacin, tylosin and tilmicosin. All isolates with MIC $>=$ $0.63 \mu \mathrm{g} / \mathrm{ml}$ for tylosin and MIC $>=1.25 \mu \mathrm{g} / \mathrm{ml}$ for tilmycosin have one of these mutations, which indicates a significant role in reducing the sensitivity of M. gallisepticum to 16-membered macrolides. Fluoroquinolones, tilmicosin, tulathromycin, chlortetracycline, doxycycline and oxytetracycline are effective against Mannheimia haemolytica and Mycoplasma, which are the main causative agents of respiratory infections in lambs. Antimicrobial resistance of Mycoplasma bovis isolates to antibacterial drugs is not high. With the exception of tilmicosin, all isolates were highly susceptible to the tested antimicrobials (oxytetracycline and florfenicol). Tilmicosin and oxytetracycline are effective in treating respiratory diseases in young calves, even if they are affected by Mycoplasma spp. Tilmicosin is more effective in eliminating the clinical signs of mycoplasmosis. Treatment of mycoplasma respiratory syndrome with tulathromycin resulted in slightly higher $(P=0.009)$ therapeutic success $(87.9 \%$ and $80 \%$, respectively) than initial treatment with enrofloxacin $(70.2 \%$ and $62.5 \%$, respectively). Animals treated with tulathromycin also received fewer follow-up treatments and increased weight gain compared to animals treated with enrofloxacin. Tulatromycin was evaluated in the treatment of pneumonia in weaning pigs intranasally inoculated with Mycoplasma hyopneumoniae. Tulatromycin was also quite effective. Use of broad-spectrum drugs, which include tilmicosin, is also
\end{abstract}

\footnotetext{
* Corresponding author: kras-bi@yandex.ru
} 
promising. Therefore, the therapeutic efficacy of the analyzed drugs used for the treatment of mycoplasmosis, both in birds and animals (cattle, small ruminants, and pigs), depends both on the drugs used and on etiological agents. A significant role in the effectiveness of treatment with certain drugs is played by the infections accompanying mycoplasmosis.

\section{Introduction}

Mycoplasmas are the cause of many pathologies, both of various species of animals and birds. Most often, mycoplasmas are the etiology of diseases in birds in Asia. Laboratory research conducted by Morrow C.C. colleagues, isolates of Mycoplasma synoviae $(n=26)$ and M. gallisepticum $(n=11)$ were isolated from 164 clinical samples collected in China, India, Indonesia, Malaysia, the Philippines, the Republic of Korea and Thailand. Most of the isolates were obtained from commercial poultry systems. To purify the pathological material, the method of membrane filtration (pore size $0.45 \mu \mathrm{m}$ ) of samples was used immediately after collection, which made it possible to spend more than a week on its transportation to the laboratory. Minimum inhibitory concentrations (MIC) of enrofloxacin, difloxacin, oxytetracycline, chlortetracycline, doxycycline, tylosin, tilmicosin, tilvalosin, tiamulin, florfenicol, lincomycin, spectinomycin and 2 combinations (spectinomycin) in bullet and lincomycin were determined by the method of microbiological administration. Elevated MICs for different antimicrobials were observed in different isolates, but appear to be largely unrelated to antimicrobial treatment histories. Overall, the results were similar to other MIC surveys around the world. As a rule, low MICs were observed for tetracyclines, tiamulin and tilvalosin. Elevated MICs of tilmicosin were observed in both M. synoviae and M. gallisepticum isolates (MIC values $>=64 \mu \mathrm{g} / \mathrm{ml}$ ), and this was observed in all isolates with high MICs of tylosin. The increase in the MIC of lincomycin was mainly associated with the increase in the MIC of tilmicosin.

\section{Methods}

The results also suggest that use of antimicrobials after vaccination against mycoplasmas may affect the resistance and efficacy of the vaccine strain (field strains were more common in herds treated after vaccination), and this area of study warrants further studies.

Study shows that isolation and MIC testing can be done from remote locations and involves providing information that will allow for more effective use of antimicrobials or other methods of controlling avian mycoplasma in chickens (e.g. live vaccines) and, therefore, more responsible use of antimicrobials from the point of view of unified health [9].

Macrolide antibiotics, including tylosin and tilmicosin, are widely used in veterinary medicine for prevention and treatment of mycoplasmosis. In vitro susceptibility testing of 50 M. gallisepticum strains isolated in Israel in the period 1997-2010 carried out by a group of scientists led by Gerchman I. showed that acquired resistance to tylosin, as well as to tilmicosin, is present in $50 \%$ of them. Moreover, $72 \%(13 / 18)$ of the strains isolated from clinical specimens since 2006 showed acquired resistance to enrofloxacin, tylosin and tilmicosin. Molecular typing of field isolates, performed using target gene sequencing (GTS), identified 13 molecular types (I-XIII). Type II was predominant until 2006, while Type X, first discovered in 2008, is currently predominant. All ten type X strains were resistant to both fluoroquinolones and macrolides, indicating selective pressure leading to clonal spread of resistance. However, this was not a unique development as resistant strains 
with different molecular types of GTS were found. Wherein, the molecular basis of the resistance of $M$. gallisepticum to macrolides was revealed.

The results obtained revealed a clear correlation between single-point mutations A2058G or A2059G in the V domain of the gene encoding 23S rRNA (rRNA, MGA_01) and acquired resistance to macrolides of $M$. gallisepticum. Definitely, all isolates with MIC $>=0.63 \mu \mathrm{g} / \mathrm{ml}$ for tylosin and MIC $>=1.25 \mu \mathrm{g} / \mathrm{ml}$ for tilmycosin have one of these mutations, which indicates a significant role in reducing the sensitivity of $M$. gallisepticum to 16-membered macrolides [5].

Infectious agalactia in goats and sheep, caused by Mycoplasma agalactiae, is an infectious disease that requires rapid diagnosis in order to reduce economic losses in milk production and lamb mortality. PCR is fast, reasonable and specific for detecting this etiological agent, which is necessary for taking preventive measures. A total of 19 cultures in modified Hayflick broth stored at $-20^{\circ} \mathrm{C}$ with $50 \%$ glycerol for up to two years, where 7 identified as Mycoplasma spp. and 12 are identified as M. agalactiae by indirect immunoperoxidase and are homologous (M. agalactiae GM139) and heterologous ( $M$. pulmonis) [4].

Infectious agalactia of small ruminants is a disease that mainly affects the mammary glands, joints and eyes. The main pathogens are Mycoplasma agalactiae in sheep and $M$. agalactiae, M. mycoides subsp. mycoides and M. capricolum subsp. capricolum in goats. In addition, $M$. putrefaciens can cause a similar clinical picture, especially in goats. Infectious agalactia occurs on all five continents and is often enzootic. Infection in affected animals and herds tends to develop chronic. Excretion of mycoplasmas, occurs mainly with milk, can continue for a long time. These insidious infections associated with the carriage of mycoplasmas in the ears of healthy animals are difficult to diagnose and control. The main mode of transmission is through the sale of carrier animals and contact during livestock transfers. Transmission within the herd occurs through contact, sucking and milking [1, 12].

\section{Results}

Bacterial respiratory infections are common in lambs. Treatment shall be aimed at combating clinical signs, as well as limiting the damage to the lungs of sick animals and requires immediate action, mainly using antimicrobial agents that are effective against pathogenic bacteria. In clinical practice, correct identification of lamb pathology is essential for appropriate treatment. Fluoroquinolones, tilmicosin, tulathromycin, chlortetracycline, doxycycline and oxytetracycline are effective against Mannheimia haemolytica and Mycoplasma, which are the main causative agents of respiratory infections in lambs. The concomitant use of non-steroidal anti-inflammatory drugs is also recommended. All lambs with clinical signs shall receive the full course of treatment. The potential value of metaphylactic treatment of clinically healthy lambs in affected herds shall be assessed on a case-by-case basis. Disease management protocols shall always include changes in herd management to address factors contributing to disease development [12].

Mycoplasma spp. are unique microorganisms associated with several diseases, including pneumonia and arthritis syndrome in animals. One of the problems in determining the role of mycoplasmas in the onset of disease is their pathogenicity. This organism can be isolated from cattle, both from clinically healthy and from sick animals. With natural infection in the field, mycoplasma pneumonia is often found as a mixed infection. In addition, observations from scientific research and clinical experience have shown that the presence of mycoplasma increases the severity of respiratory illness.

There are no pathognomonic signs of mycoplasma infection. Clinical signs associated with respiratory infections include tachypnea, shortness of breath, discharge from the eyes and nose, depression, decreased appetite, bowing posture, and fever. Clinical signs 
associated with joint infections include stiffness, lameness, difficulty standing up, swollen joints and tendon sheaths, decreased appetite, and weight loss.

This microorganism for cultivation requires special nutrient media and special conditions for cultivation in the laboratory. If the practicing veterinarian wants to confirm the diagnosis by culture, then when sending samples to the laboratory, it is necessary to request culture for mycoplasma. Besides determining the meaning of Mycoplasma spp. in the case of an illness, the practitioner shall obtain laboratory advice on use of appropriate and effective drug therapies.

Therapy for mycoplasmosis, both experimentally and in the field, is not always unambiguous and often does not bring results. As mycoplasmas are resistant to multiple treatments, robust biosecurity and biosecurity programs that minimize stress and exposure to the body are the best guidelines that clinicians can discuss with manufacturers for disease prevention and control [14].

The effects of antimicrobial therapy and prevention strategies on respiratory disease in bovine animals, as well as the genetic relationship and antimicrobial resistance of Mycoplasma bovis isolates in feeding in western Canada, were studied by Hendrick S.H. and colleagues. Calves of the feedlot $(n=3784)$ received oxytetracycline $($ OTC) as treatment or without use of an antimicrobial drug, as well as florfenicol according to the scheme: once subcutaneously or twice intramuscularly (with an interval of 48 hours), if a diagnosis was made of bovine respiratory disease (BRD) caused by Mycoplasma bovis. Calves from different treatment groups were pooled and observed until re-implantation (similar to 100 days). Animals receiving OTC drugs had a reduced risk of BRD, an increased risk of arthritis, and no significant difference in mean daily gain, BRD relapse, overall mortality or BRD mortality. There were no significant differences between treatment protocols. Deep nose swabs $(n=233)$ taken on arrival $(n=122)$, treatment $(n=$ $77)$, and dissection swabs from lungs and joints $(n=34)$ were cultured on Mycoplasma bovis from 61 animals, only, that sick or dying from a chronic course of the disease (pneumonia and arthritis) and from 61 healthy calves. There were significant differences in isolate diversity $(\mathrm{n}=51)$ between study years and different cattle populations. Antimicrobial treatment did not affect isolate diversity. With the exception of tilmicosin, the isolates were highly susceptible to the tested antimicrobial agents [6].

\section{Discussion}

In veterinary practice, the most popular is use of prolonged-release drugs. Comparison of tilmicosin with long-acting oxytetracycline in the treatment of respiratory diseases in calves was studied by Musser, J. with colleagues. The aim of the experiment was to compare the effect of a single parenteral injection of tilmicosin with the effect of a single dose of longacting oxytetracycline as a treatment in the early stages of natural undifferentiated airway disease in young dairy calves.

The experiment involved 40 dairy-aged calves from 5 farms, which were examined weekly up to 3 months of age. When the veterinarian diagnosed a respiratory illness, the calves were assigned to one of two treatment groups. Transtracheal lavage samples were taken to characterize the pathogens. The veterinarian, who did not know about the appointment of the treatment, examined the calves within 3 days after the treatment and assessed the severity of the course of the disease using a scoring system. The growth rates were measured.

As a result of the experiments, it was found that the body's response to initial treatment, the frequency of relapses and the effect on the growth rate of antibiotics were found to be equally effective. The severity of clinical disease was significantly less $(\mathrm{P}<0.03)$ in those treated with tilmicosin on the 2 nd and 3rd days after the start of treatment. Analysis of 
transtracheal wash swabs showed Pasteurella multocida - from 25 out of 40 calves, P. haemolytica (4/40), Haemophilus somnus (4/40), Actinomyces pyogenes (3/40) and Aspergillus spp. (2/40). Mycoplasma was isolated together with bacterial isolates from 22 calves.

Experiments have shown that tilmicosin and oxytetracycline are effective in treating respiratory diseases in young calves, even if they are affected by Mycoplasma spp. Tilmicosin is more effective in eliminating the clinical signs of mycoplasmosis. Early treatment of dairy calves with respiratory diseases can reduce the negative impact on their growth and development [10].

The efficacy of tulathromycin versus enrofloxacin for the initial treatment of natural respiratory disease in fattening cattle was studied by Robb E.J. and colleagues. After the arrival of clinically bovine respiratory disease (BRD) calves for rearing at one of the feedlots, the calves were randomly assigned to be treated with tulathromycin $(2.5 \mathrm{mg} / \mathrm{kg} \mathrm{sc})$ or enrofloxacin $(12.5 \mathrm{mg} / \mathrm{kg} \mathrm{sc})$. Additional administration of the drug to calves that did not respond to the initial treatment was done according to the prescribed course. Initial treatment with tulathromycin resulted in slightly higher $(\mathrm{P}=0.009)$ therapeutic success ( $87.9 \%$ and $80 \%$, respectively) than initial treatment with enrofloxacin $(70.2 \%$ and $62.5 \%$, respectively). Animals treated with tulathromycin also received fewer follow-up courses and higher weight gain than animals treated with enrofloxacin [13].

Evaluation of use of tulathromycin for treatment of pneumonia after experimental infection of pigs with Mycoplasma hyopneumoniae was carried out by McKelvie $J$ and colleagues. Tulatromycin was evaluated in the treatment of pneumonia in weaning pigs intranasally inoculated with Mycoplasma hyopneumoniae. Five days post infection, pigs were randomized to be treated with a single intramuscular injection of saline, a single intramuscular injection of tulathromycin $(2.5 \mathrm{mg} / \mathrm{kg}$; day 0$)$, or three intramuscular injections of enrofloxacin $(5.0 \mathrm{mg} / \mathrm{kg}$; days $0,1,2)$. The pigs were necropsied on day $12 \mathrm{or}$ 13. Uninfected control animals remained healthy without lung pathology. Compared with saline, cough, mean lung injury score and proportional lung weight were significantly reduced, and weight gain was significantly greater in pigs treated with tulathromycin ( $\mathrm{P}$ $<0.05)$. Compared to enrofloxacin, there were no significant differences in proportional lung weight or weight gain, but rates of cough and lung damage were higher in pigs treated with tulathromycin $(\mathrm{P}<0.05)$. Therefore, it can be stated that tulatromycin was effective in the treatment of pneumonia after experimental infection with $\mathrm{M}$. hyopneumoniae [8].

A study of the efficacy and safety of tilmicosin phosphate in the treatment of experimental mycoplasma infections in pigs was carried out by Zhang, X.H. et al. To evaluate the therapeutic effect of oral administration of tilmicosin phosphate on mycoplasma pneumoniae in pigs and its safety in infected pigs, a model of pig mycoplasma pneumoniae disease by artificial infection was created. We analyzed such indicators as efficiency, cure rate. Mortality rates, lung injury scores, standard blood tests, blood biochemistry and standard porcine urine tests were determined. The results showed that all dosage groups: $100 \mathrm{mg} / \mathrm{L}, 80 \mathrm{mg} / \mathrm{L}, 60 \mathrm{mg} / \mathrm{L} 10 \%$ soluble tilmicosin phosphate powder showed clear therapeutic effects on mycoplasma pneumonia in pigs, which significantly reduced the number of lung lesions in patients, and that the therapeutic the effect of soluble tilmicosin phosphate powder at a dose of $60-80 \mathrm{mg} / \mathrm{l}$ was equivalent to the effect of $10 \%$ soluble tilmicosin powder. Administration of $10 \%$ powder, soluble tilmicosin phosphate, improved weight gain in sick pigs. Treatment of infected pigs at a dose of 60-100 mg/L of $10 \%$ soluble powder, tilmicosin phosphate, did not affect the standard blood test results, biochemical index and standard urinalysis performed in a test mode, and, therefore, is safe for sick pigs. In conclusion, it shall be noted that the introduction of $10 \%$ soluble powder of tilmicosin phosphate is effective and safe for the treatment of artificially infected pigs with the causative agent of mycoplasma pneumonia [15]. 
Clark L.K. and colleagues evaluated the efficacy of the macrolide antibiotic in reducing the number of respiratory pathogens in piglets weaned from sows at 12 and 21 days. The aim of their study was to determine the therapeutic effect of a feed antibiotic (tilmicosin) on pigs infected with Mycoplasma hyopneumoniae, and to find out if the status of $\mathrm{M}$. hyopneumoniae in pigs weaned from a sow at 21 days of age using tilmicosin, similar to $\mathrm{M}$. hyopneumoniae in pigs, weaned from the sow 12 days after birth. The researchers further evaluated the effect of tilmicosin on other respiratory pathogens in all treated piglets.

The scheme of the method is as follows. Fifty commercial pigs were randomly divided into five experimental groups. Three groups were weaned from a sow at 12 days of age and were either 1) infected with M. hyopneumoniae and treated with tilmicosin (treated with CEW), 2) infected but not treated with tilmicosin (CEW untreated), or 3) left untreated and untreated (control EW). Two groups were weaned from sows at 21 days of age, were not challenged with M. hyopneumoniae, and were either treated (treated with LW) or not treated (control LW) with tilmicosin. Some pigs in all treatment groups the clinical signs similar to Haemophilus parasuis disease developed and they were treated with penicillin for 3 consecutive days immediately prior to infection of the two groups of early weaning pigs. The weight gain of each pig was measured as the difference in weight between 12 and 56 days of age. The presence of respiratory disease was taken into account by the number of pigs in which cough was observed every day, and by the scores of lung damage at autopsy. Pigs were sacrificed at 56 days of age and examined for M. hyopneumoniae, Actinobacillus pleuropneumoniae, H. parasuis, Pasteurella multocida, Streptococcus suis and Bordetella bronchiseptica. In addition, sera from all pigs were evaluated for antibodies to $\mathrm{M}$. hyopneumoniae and A. pleuropneumoniae when the pigs were 12 and 56 days old.

As a result, it was found that tilmicosin did not affect the growth rate of different groups of pigs. Tilmicosin reduced $(\mathrm{P}<0.01)$ cough in pigs treated with $\mathrm{CEW}$. Although lung injury rates in pigs receiving $\mathrm{CEW}$ were not significantly lower than in pigs in the group not receiving $\mathrm{CEW}(\mathrm{P}>0.05)$, tilmicosin appeared to delay the onset of infection during treatment. Actinobacillus pleuropneumoniae, B. bronchiseptica and P. multocida were not isolated from any pig tissue. Four of the seven pigs from which S. suis was isolated were from the LW control group, while S. suis was not isolated from any of the LW treated pigs. Haemophilus parasuis was isolated by necropsy from 26 pigs in this trial: 19 out of 20 LW pigs and 7 out of 30 early weaning pigs.

In the age groups of weaning, tilmicosin did not affect the likelihood of isolating the $\mathrm{H}$. parasuis culture. Pigs in all five groups were seropositive for A. pleuropneumoniae at 12 days of age, but titers decreased during the experiment. Two out of ten LW control pigs seroconverted to $M$. hyopneumoniae during the experiment, whereas none of the LW treated pigs or EW control pigs seroconverted.

Therefore, tilmicosin did not reduce mycoplasma pneumoniae lesions when added to the feed of CEW-fed pigs. Tilmicosin delayed the onset of coughing and thus probably delayed the development of lung pathology while given to pigs receiving CEW. Tilmicosin decreased S. suis colonization and M. hyopneumoniae seroconversion in LW pigs [3].

Mycoplasmosis often occurs in the presence of other infectious agents. Use of drugs with a wide spectrum of action, which includes tilmicosin, is promising. The aim of the studies by MacInnes, J.I. and colleagues was to determine the effectiveness of prophylactic use of tilmicosin in the control of experimentally induced Haemophilus parasuis infection in pigs. The scientists took into account the indicators of the influence of tilmicosin introduced into the feed on the morbidity, mortality and productive parameters of pigs experimentally infected with the virulent field strain of Haemophilus parasuis.

The study consisted of three replicate trials in which pigs aged 19 to 22 days were randomly assigned to one of four treatment groups (nine pigs per group): no treatment - no treatment, with infection - no treatment, infected and sanitized tilmicosin $200 \mathrm{mg}$ per $1 \mathrm{~kg}$ 
of body weight and infected and treated with tilmicosin, at a dose of $400 \mathrm{mg}$ per $\mathrm{kg}$ of body weight. The test groups were randomly assigned to one of three cells. The untreated group was placed in the same cage for three repetitions to minimize the possibility of crosscontamination with $\mathrm{H}$. parasuis. The medicated food was given from day 0 until the end of the trial (day 21). Infection was carried out with an aerosol of a virulent isolate of serotype $5 \mathrm{H}$. parasuis on day 7. Outcomes assessed included mortality, gross lesions, and postmortem H. parasuis culture, as well as clinical assessments and growth parameters.

Results: among infected pigs, compared to untreated pigs, treatment with tilmicosin at a dose of $200 \mathrm{mg} / \mathrm{kg}$ feed reduced the incidence of severe injuries consistent with $\mathrm{H}$. parasuis infection, as well as $\mathrm{H}$. parasuis shedding and mortality rates specific to $\mathrm{H}$ parasuis $(\mathrm{P}$ $<0.05)$, as well as clinical indicators of recovery and growth parameters $(\mathrm{P}<0.05)$, excluding average daily gain. In addition, a significant trend $(\mathrm{P}<0.05)$ for further improvement in all parameters with increasing tilmicosin dose $(400 \mathrm{mg} / \mathrm{kg})$ was demonstrated by linear regression.

Therefore, under the conditions of this study, tilmicosin was effective against $\mathrm{H}$. parasuis infection in weaning pigs infected by aerosol inoculation of a virulent field strain [7].

The effect of tilmicosin on minimizing the occurrence of atrophic rhinitis, pneumonia and pleurisy in pigs was studied by Olson L.B. and Backstrom L.R.

To achieve this goal, they carried out the prophylaxis of rearing piglets receiving tilmicosin for 3 weeks from progressive atrophic rhinitis (PAR) when kept together with pigs with clinical manifestations of this disease.

Study Methods. In the experiments, recipient pigs were used in contact with donor pigs that showed clinical signs of PAR and did not receive tilmicosin (NME group), the second group (ME group) received tilmicosin with food for 3 weeks; the third control group was unaffected and did not receive medication. Pig weights and nasal swabs for bacterial culture were collected on study days 0,21 and 42. Feed intake was also monitored. On day 21 of the study, all drugs were removed from the feed (ME) and the donor animals were sent for postmortem examination. On the 42nd day of the study, the pigs of the NME, ME and control groups were sacrificed and subjected to postmortem examination. The shape of the muzzle, the presence of pneumonia and pleurisy were assessed in points using a program for processing and analyzing digital images.

As a result, overall body weight gain was higher in the control pigs than in the ME or NME pigs. Average daily weight gain and feed intake were significantly higher $(\mathrm{P}<0.05)$ in ME pigs compared to NME pigs throughout the study. The efficacy of feed intake did not differ significantly between groups. All recipient pigs had negative laboratory culture for Bordetella bronchiseptica and Pasteurella multocida on day 0. Control pigs remained negative for P. multocida types A and D throughout the study, and only one pig was positive for B. bronchiseptica when cultured at the end of the study. A higher percentage of NME pigs than ME pigs were positive for P. multocida shedding on days 21 and 42 . Starting from 21-42 days of the study, there was a marked increase in the number of positive isolates of $\mathrm{P}$. multocida type $\mathrm{A}$ in pigs of the ME group. At slaughter, control pigs showed no serious pneumonia lesions and had lower visual muzzle scores compared to pigs in the ME and NME groups. Visual atrophic rhinitis scores were significantly higher (P $<0.05$ ) in NME pigs compared to ME pigs.

Therefore, treatment with tilmicosin led to a decrease in the indices of changes in muzzle shape and better growth in the ME pigs than in the NME pigs [11]. 


\section{Conclusion}

Mycoplasmas are the cause of many pathologies, both of various species of animals and birds. Minimum inhibitory concentrations (MICs) of enrofloxacin, difloxacin, oxytetracycline, chlortetracycline, doxycycline, tylosin, tilmycosin, tilvalosin, tiamulin, florfenicol, lincomycin, spectinomycin and the 2 combinations (spectinomycin and lincomycin) with isolates of Mycoplasma synoviae and M. gallisepticum differed significantly. Elevated MICs of tilmicosin were observed in both M. synoviae and $M$. gallisepticum isolates (MIC values $>=64 \mu \mathrm{g} / \mathrm{ml}$ ), and this was observed in all isolates with high MICs of tylosin. The increase in the MIC of lincomycin was mainly associated with the increase in the MIC of tilmicosin. In vitro susceptibility testing of $50 \mathrm{M}$. gallisepticum strains isolated in Israel in the period 1997-2010 carried out by a group of scientists led by Gerchman I. showed that acquired resistance to tylosin, as well as to tilmicosin, is present in $50 \%$ of them. Moreover, $72 \%(13 / 18)$ of the strains isolated from clinical specimens since 2006 showed acquired resistance to enrofloxacin, tylosin and tilmicosin. All isolates with MIC $>=0.63 \mu \mathrm{g} / \mathrm{ml}$ for tylosin and MIC $>=1.25 \mu \mathrm{g} / \mathrm{ml}$ for tilmycosin have one of these mutations, which indicates a significant role in reducing the sensitivity of $M$. gallisepticum to 16-membered macrolides. Fluoroquinolones, tilmicosin, tulathromycin, chlortetracycline, doxycycline and oxytetracycline are effective against Mannheimia haemolytica and Mycoplasma, which are the main causative agents of respiratory infections in lambs. Antimicrobial resistance of Mycoplasma bovis isolates to antibacterial drugs is not high. With the exception of tilmicosin, all isolates were highly susceptible to the tested antimicrobials (oxytetracycline and florfenicol). Tilmicosin and oxytetracycline are effective in treating respiratory diseases in young calves, even if they are affected by Mycoplasma spp. Tilmicosin is more effective in eliminating the clinical signs of mycoplasmosis. Treatment of mycoplasma respiratory syndrome with tulathromycin resulted in slightly higher $(\mathrm{P}=0.009)$ therapeutic success $(87.9 \%$ and $80 \%$, respectively) than initial treatment with enrofloxacin $(70.2 \%$ and $62.5 \%$, respectively). Animals treated with tulathromycin also received fewer follow-up treatments and increased weight gain compared to animals treated with enrofloxacin. Tulatromycin was evaluated in the treatment of pneumonia in weaning pigs intranasally inoculated with Mycoplasma hyopneumoniae. Tulatromycin was quite effective. Use of broad-spectrum drugs, which include tilmicosin, is also promising. Tilmicosin was effective against $H$. parasuis infection in weaned pigs.

Therefore, the therapeutic efficacy of the analyzed drugs used for the treatment of mycoplasmosis, both in birds and animals (cattle and small ruminants and pigs), depends both on the drugs used and on the etiological agents. A significant role in the effectiveness of treatment with certain drugs is played by the infections accompanying mycoplasmosis.

\section{References}

1. D. Bergonier, X. Berthelot, F. Poumarat, Revue scientifique et technique de 1 office international des epizooties, 16, 3 (1997)

2. D. Bergonier, F. Poumarat, Revue scientifique et technique de 1 office international des epizooties, 15, 4 (1996)

3. L.K. Clark, C.C. Wu, A. Van, W.G . Van Alstine, K.E. Knox, Swine health and production, 6, 6 (1998)

4. J.F. De Almeida, E.R. do Nascimento, V.L. de Almeida Pereira, M.L. Barreto, C.A. de Martino Campos, E.O. de Azevedo, Revista brasileira de medicina veterinaria, 29, 2 (2007) 
5. I. Gerchman, S. Levisohn, I. Mikula, L. Manso-Silvan, I. Lysnyansky, Veterinary research, 42 (2011)

6. S.H. Hendrick, K.G. Bateman, L.B. Rosengren, Canadian veterinary journal-revue veterinaire canadienne, 54, 12 (2013)

7. J.I. MacInnes, M.A. Paradis, G.H. Vessie, L. Slavic, S. Watson, J.B. Wilson, J.J. Aramini, C.P. Dick, Journal of swine health and production, 11, 4 (2003)

8. J. McKelvie, J.H. Morgan, I.A. Nanjiani, J. Sherington, T.G. Rowan, S.J. Sunderland, Veterinary the rapeutics, 6, 2 (2005)

9. C.J. Morrow, Z. Kreizinger, R.R. Achari, K. Beko, C. Yvon, M. Gyuranecz, Veterinary microbiology, 250 (2020)

10. J. Musser, G.D. Mechor, Y.T. Grohn, E.J. Dubovi, S. Shin, Journal of the american veterinary medical association, 208, 1 (1996)

11. L.B. Olson, L.R. Backstrom, Swine health and production, 8, 6 (2000)

12. A.P. Politis, N.G.C. Vasileiou, K.S. Ioannidi, V.S. Mavrogianni, Small ruminant research, 176 (2019)

13. E.J. Robb, C.M. Tucker, L. Corley, W.L. Bryson, K.C. Rogers, K. Sturgess, D.J. Bade, B. Brodersen, Veterinary therapeutics, 8, 2 (2007)

14. D.L. Step, J.G. Kirkpatrick, Bovine practitioner, 35, 2 (2001)

15. X.H. Zhang, J.Z. Pan, N. Wu, S. Tang, X.D. Lei, Y.Y. Sun, J. Hartung, E.D. Bao, Turkish journal of veterinary \& animal sciences, 42, 6 (2018)

16. A. Mecke, I. Lee, J.R. Baker jr., M.M. Banaszak Holl, B.G. Orr, Eur. Phys. J. E 14, 7 (2004)

17. M. Ben Rabha, M.F. Boujmil, M. Saadoun, B. Bessaïs, Eur. Phys. J. Appl. Phys. (to be published)

18. F. De Lillo, F. Cecconi, G. Lacorata, A. Vulpiani, EPL, 84 (2008)

19. L. T. De Luca, Propulsion physics (EDP Sciences, Les Ulis, 2009) 